\title{
Spatial and temporal characteristics of electrically fixed holograms in photorefractive strontium-barium niobate
}

\author{
Sergei Orlov and Demetri Psaltis \\ California Institute of Technology, Department of Electrical Engineering 116-81, \\ Pasadena, California 91125 \\ Ratnakar R. Neurgaonkar \\ Rockwell International Science Center, 1049 Camino dos Rios, Thousand Oaks, California 91360
}

(Received 18 October 1993; accepted for publication 9 December 1993)

\begin{abstract}
We report measurements of the holographic diffraction efficiency (HDE) of single photorefractive gratings fixed by an externally applied voltage pulse in $\mathrm{Sr}_{0.75} \mathrm{Ba}_{0.25} \mathrm{Nb}_{2} \mathrm{O}_{6}$. The polarization grating HDE and decay rate are strongly dependent on the grating spacing and also depend on the intensity of the light beams during the recording and erasing/reconstruction process. Knowledge of the dependence of the fixing process on spatial frequency may be useful for the creation of physical models of local polarization switching in ferroelectrics.
\end{abstract}

Several procedures of fixing of volume-phase holograms in photorefractive materials have been demonstrated in the past and recently. Thermal fixing ${ }^{1-4}$ involves the formation of a complementary ionic grating at elevated temperature with subsequent cooling of the crystal. Hologram fixation, which involves ferroelectric polarization and periodic domain structure formation, was achieved by applying a voltage pulse after the recording, ${ }^{5-7}$ by high light intensity writing, ${ }^{8,9}$ with the use of screening of externally applied voltage, ${ }^{10}$ and also via cooling through ferroelectric phase transition. ${ }^{11,12}$ However, a quantitative model describing the periodic and pseudorandom ferroelectric microdomain structure formation is yet to be proposed.

In this letter we report the results of our studies on electrical fixing of holograms in $\mathrm{Sr}_{0.75} \mathrm{Ba}_{0.25} \mathrm{Nb}_{2} \mathrm{O}_{6}$ (SBN:75). The crystal sample characterization and optical setup description appears in Ref. 7. Ordinarily polarized expanded beams are used for recording and the holographic diffraction efficiency (HDE) is monitored with a low-power extraordinarily polarized $\mathrm{He}-\mathrm{Ne}$ laser beam incident at the Bragg angle. Fixing is achieved by applying a negative (opposite to the direction of spontaneous polarization) voltage pulse in the dark, i.e., after the recording beams are blocked. After the voltage pulse the initial photorefractive grating is erased by a uniform non-Bragg-matched ordinarily polarized beam. After the HDE reaches steady state, a strong positive (along the initial direction of spontaneous polarization) voltage pulse is applied in order to erase the polarization grating and to reveal the optically erasable compensating component. ${ }^{7,13}$ A typical cycle of recording, fixation, optical erasure, polarization grating decay, and electrical revealing is shown in Fig. 1.

After each fixing experiment the crystal is poled by applying strong positive voltage $(\simeq 2.5 \mathrm{kV})$ along the $c$ axis for 20-30 min at room temperature. No fixing was achieved (with these recording time and light intensities) if the negative voltage pulse was not applied. No significant degradation in the optical quality of the crystal and the HDE of the ordinary photorefractive gratings is observed due to the re- peated fixing cycles throughout the whole series of fixing experiments.

Two series of experiments with different light intensities (low: $I_{\text {writing }} \simeq 4 \mathrm{~mW} / \mathrm{cm}^{2}, \quad I_{\text {crasing }} \approx 8 \mathrm{~mW} / \mathrm{cm}^{2}$; high: $I_{\text {writing }} \simeq 35 \mathrm{~mW} / \mathrm{cm}^{2}, I_{\text {erasing }} \simeq 70 \mathrm{~mW} / \mathrm{cm}^{2}$ ) have been carried out. The only parameter that was changed during each series is the grating spacing $\Lambda$, which is determined by the angle of the recording beams inside the crystal.

The dependence of the fixed hologram HDE [(F) in Fig. 1] on the grating spacing is presented in Fig. 2. The linear fits on a $\log -\log$ scale at high spatial frequencies, where the effect of compensation ${ }^{13}$ is small, have slopes $2.1 \pm 0.2$ (low intensity) and 1.3 \pm 0.1 (high intensity). This suggests a relationship $\eta^{\text {fixed }} \propto \Lambda^{1.3} \div \Lambda^{2.1}$ for the fixed polarization grating HDE for small values of $\Lambda$ (in fact, for $\Lambda \leqslant \Lambda_{e}$, where $\Lambda_{e}$-Debye screening length ${ }^{13}$, which, for this crystal, was

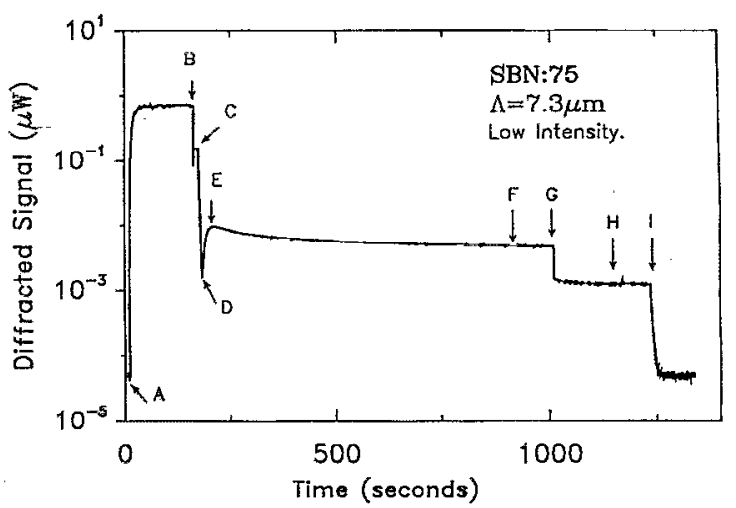

FIG. 1. Diffracting signal as a function of time during the fixing experiment with low intensity. Fixing pulse amplitude is $-1650 \mathrm{~V} / \mathrm{cm}$ and its duration $0.5 \mathrm{~s}$. Recording beams intensities ratio $m \approx 1, \lambda=488 \mathrm{~nm}$. The transmitted probe light power without the grating is $2.3 \mu \mathrm{W}$. A: Recording begins. $\mathrm{B}$ : Writing beams are blocked and a negative voltage pulse is applied. C: Optical erasure with a non-Bragg-matched beam begins. $D$ : The transient dip due to $180^{\circ}$ phase shift between the polarization and the initial photorefractive gratings. E: Peak in HDE of the fixed grating; slow decay of fixed grating begins. F: Steady state of the fixed grating. G: Positive voltage pulse ( $E=4.2 \mathrm{kV} / \mathrm{cm}$, duration $2 \mathrm{~s}$ ) is applied (erasing beam is blocked). $\mathrm{H}$ : Revealed compensating grating. I: Optical erasure. 


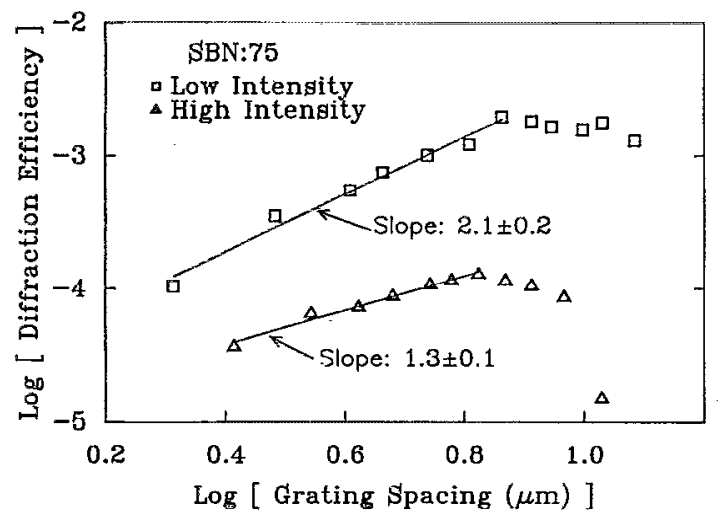

FIG. 2. Log-log plot of the fixed (steady state) holograms HDEs [(F) in Fig. $1]$ vs grating spacing $\Lambda$. From the linear fits: for small $\Lambda$ 's $\eta^{\text {fixed }} \propto \Lambda^{2.1 \pm 0.2}$ (low intensities); $\eta^{\text {fixed }} \propto \Lambda^{1,3 \pm 0,1}$ (high intensities).

approximately $12.5 \pm 0.5 \mu \mathrm{m}$ ). We believe that the falloff of $\eta^{\text {fixed }}$ at large grating periods is primarily due to increasing compensation of the polarization hologram by a dynamic grating, ${ }^{13}$ and also because of the decrease in the photorefractive response of the crystal for large spatial periods (namely, when $\Lambda \geqslant \Lambda_{e}$ ).

We also measured the HDE of the optically erasable compensating grating $\eta^{\text {comp }}[(\mathrm{H})$, Fig. 1$]$, which reveals itself after electrical erasure of the polarization hologram. These results are shown in Fig. 3. In agreement with the model proposed in Ref. 13, the compensating grating HDE grows very fast with $\Lambda$. The experimental data suggest that for small $\Lambda$ 's $\left(\Lambda \leqslant \Lambda_{e}\right.$, where the compensating grating is much weaker than the fixed grating) $\eta^{\text {comp }} \propto \Lambda^{5.5} \div \Lambda^{6}$ (at the same time: $\eta^{\text {comp }} / \eta^{\text {fixed }} \propto \Lambda^{4}$, even in the region where $\eta^{\text {comp }}$ is comparable or larger than $\eta^{\text {fixed }}$, Ref. 13).

It should be noted, however, that the HDEs of fixed and revealed compensating holograms appeared to be dependent on writing and/or erasing beam intensities. This effect cannot be understood in the framework of a model that accounts only for the formation of the space-charge field. ${ }^{13}$ The microdomain formation due to biased (or unbiased) internal photorefractive space-charge field and their subsequent de-

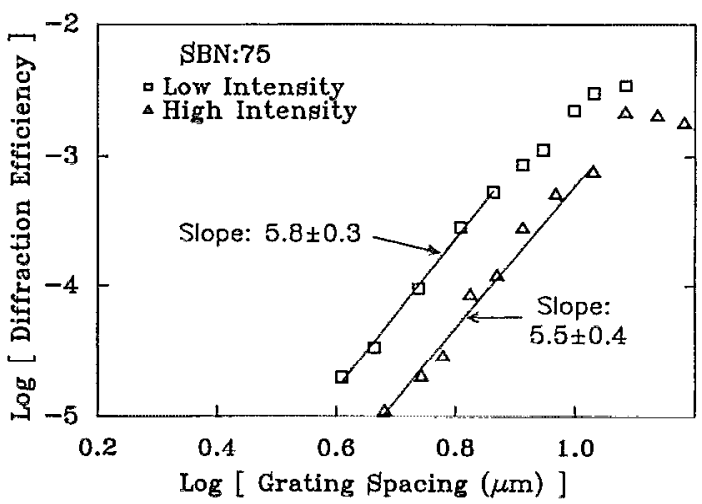

FIG. 3. Log-log plot of the diffraction efficiency of the compensating gratings [(H) in Fig. 1] revealed by the positive poling pulse. From the linear fits: For sufficiently small grating spacings $\left(\Lambda \leqslant \Lambda_{e}\right) \eta^{\text {comp } \propto \Lambda^{5.8 \pm 0.3}}$ (low intensities); $\eta^{\text {comp } \propto} \Lambda^{5.5 \pm 0.4}$ (high intensities).

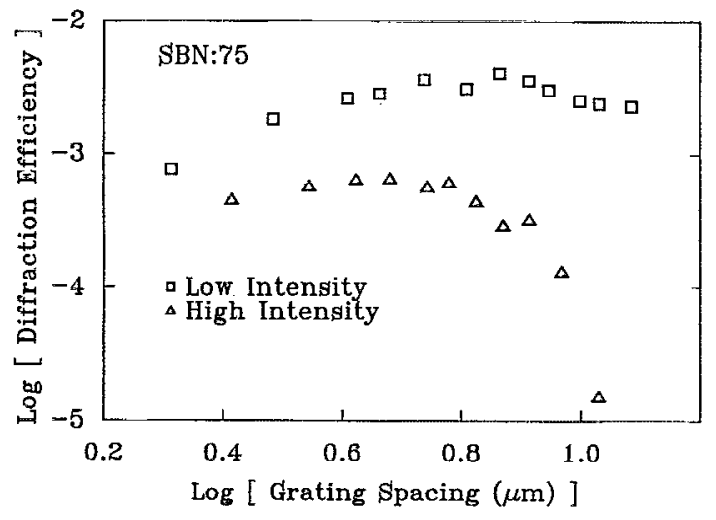

FIG. 4. $\log -\log$ plot of the peak value of the fixed grating HDEs [(E) in Fig. 1] vs grating spacing $\Lambda$.

cay to the equilibrium state must also be taken into account.

The transition between the application of the fixing pulse [(B), Fig. 1] and the steady state of the fixed hologram [(F), Fig. 1] has two significant features. The first is the transient dip [(D), Fig. 1] due to $180^{\circ}$ phase shift between the initial photorefractive and the polarization gratings. The second is the peak in HDE [(E), Fig. 1] with subsequent decay to the steady state. In Fig. 4 we plot the peak value of the polarization hologram HDE versus grating spacing for two different intensities. Unlike fixing via high intensity recording, ${ }^{9}$ in our experiments both the peak and the steady-state values of the fixed grating HDEs decrease with increasing light intensity. Therefore, for the type of fixing discussed here, both the recording and the readout should be performed with relatively low intensity beams. This also reduces the amplitude of the pyroelectric and thermal gratings ${ }^{14}$ in the volume of the crystal, which can be comparatively large in the vicinity to the ferroelectric phase transition temperature of the crystal $\left(T_{c}=55^{\circ} \mathrm{C}\right.$ for SBN:75).

The ratio of the peak HDE [(E), Fig. 1] and the steadystate value $[(\mathrm{F})$, Fig. 1] is shown in Fig. 5. As can be seen, holograms with smaller spatial period appear to be less stable and are subject to stronger decay. This decay of polarization gratings suggests that the equilibrium (or quasiequilibrium

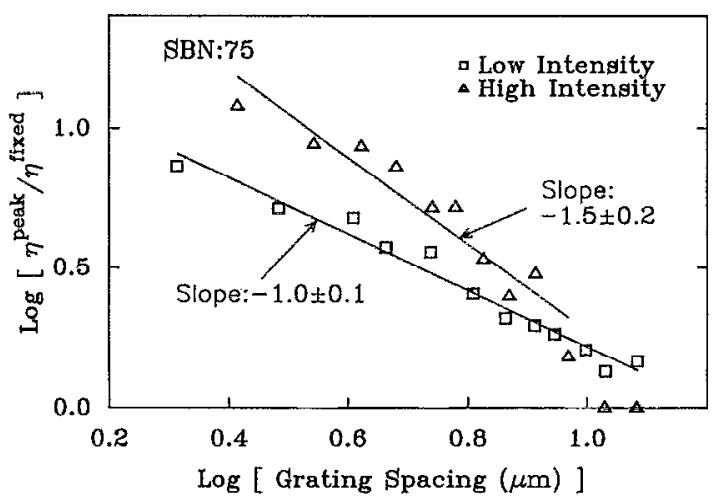

FIG. 5. Log-log plot of the ratio of the peak [(E) in Fig. 1] and the steadystate [(F) in Fig. 1] values of HDEs of the fixed grating vs grating spacing. From the linear fits: $\eta^{\text {peak }} / \eta^{\text {fixed }} \propto \Lambda^{-1.0 \pm 0.1}$ (low intensities); $\eta^{\text {peak }} / \eta^{\text {fixed }} \propto \Lambda^{-1.5 \pm 0.2}$ (high intensities). 


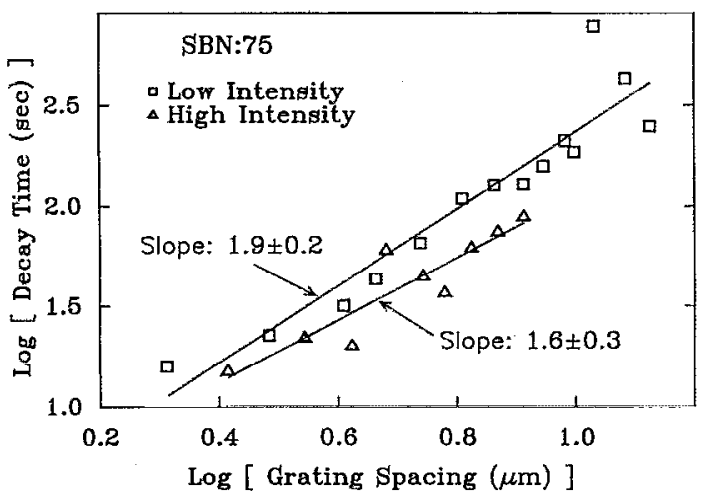

FIG. 6. Log-log plot of the polarization modulation decay time constant vs grating spacing. Values of $\tau_{\text {decay }}$ were obtained by fitting the experimental curves of time dependence of HDE $\eta(t)$ with $\sqrt{\eta(t)}=\exp \left[-\left(t-t_{0}\right) / \tau_{\text {decay }}\right]$ in the region from the peak in HDE [(E) in Fig. 1] to the steady state [(F) in Fig. 1]; $t_{0}$-normalization parameter, which does not affect the value of $\tau_{\text {decay }}$. From the linear fits: $\tau_{\text {decay }} \propto \Lambda^{1.9 \pm 0.2}$ (low intensities); $\tau_{\text {decay }} \propto \Lambda^{1.6 \pm 0.3}$ (high intensities).

with a long lifetime) value of $\eta^{\text {fixed }}$ may be largely dictated by the microdomain dynamics and their thermodynamic stability, rather than by the experimental parameters that we can control. Recent studies, ${ }^{15}$ which revealed the effect of saturation in fixed HDE with respect to the fixing pulse amplitude, support this hypothesis.

Assuming that the amplitude of the polarization modulation $P_{s_{1}}$ decays exponentially, and $\eta^{\text {fixed }} \propto\left(P_{s_{1}}\right)^{2}$ (Ref. 13), we evaluate the decay time constants of polarization holograms from peak value to the steady state. These results are represented in Fig. 6. Gratings with high spatial frequencies decay faster. The dependence suggested by the data obtained is $\tau_{\text {decay }} \propto \Lambda^{1.6} \div \Lambda^{2}$. It should be noted that the strong dependence of the decay rate on the spatial period is significant since it implies that the size and the geometry of the microdomains being formed depends not only on the local amplitude of the electric field, but also on its spatial frequency. We have no obvious explanation for such long-range interac- tions, other than that the longitudinal extent of the microdomains, which are responsible for the grating formation, is comparable with the grating spacing itself $(0.5-10 \mu \mathrm{m})$. The transversal size, however, may be very small; of the order of several unit cells.

In conclusion, we presented experimental results of electrical fixing in SBN:75. The HDE of the fixed grating is dependent on the spatial period with a maximum around the Debye screening length, and also depends on the intensity of the light beams. Our experiments suggest that the steadystate HDE most likely is dictated by microdomain thermodynamic stability, rather than by the parameters of the fixing techniques. Gratings with higher spatial frequencies are subject to stronger and faster decay than those with lower ones. The strong dependence of the polarization grating decay rate on $\Lambda$ suggests that the longitudinal size of microdomains, responsible for the fixed hologram formation, is of the order of its grating spacing $(0.5-10 \mu \mathrm{m})$.

This work was supported by the Advanced Research Project Agency and the U. S. Air Force Office of Scientific Research. We thank Yong Qiao, Jiafu Luo, and Whye-Kei Lye for their help.

${ }^{1}$ J. J. Amodei and D. L. Staebler, Appl. Phys. Lett. 18, 540 (1971).

${ }^{2}$ L. Arizmendi, J. Appl. Phys. 65, 423 (1989).

${ }^{3}$ G. Montemezzani and P. Gunter, J. Opt. Soc. Am. B 7, 2323 (1990).

${ }^{4}$ D. Kirillov and J, Feinberg, Opt. Lett. 16, 1520 (1991).

${ }^{5}$ F. Micheron and G. Bismuth, Appl. Phys. Lett. 20, 79 (1972).

${ }^{6}$ F. Micheron and G. Bismuth, Appl. Phys. Lett. 23, 71 (1973).

${ }^{7}$ Y. Qiao, S. Orlov, D. Psaltis, and R. Neurgaonkar, Opt. Lett. 18, 1004 (1993).

${ }^{8}$ J. B. Thaxter and M. Kestigian, Appl. Opt. 13, 913 (1974).

${ }^{9}$ A. Kewitsch, M. Segev, A. Yariv, and R. Neurgaonkar, Opt. Lett. 18, 1262 (1993).

${ }^{10}$ M. Horowitz, A. Bekker, and B. Fischer, Appl. Phys. Lett. 62, 2619 (1993).

${ }^{11}$ F. Micheron and J. Trotier, Ferroelectrics 8, 441 (1974).

${ }^{12}$ V. Leyva, A. Agranat, and A. Yariv, Opt. Lett. 16, 554 (1991).

${ }^{13}$ S. Orlov, D. Psaltis, and R. Neurgaonkar, Appl. Phys. Lett. 63, 2466 (1993).

${ }^{14}$ K. Buse, J. Opt. Soc. Am. B 10, 1266 (1993).

${ }^{15}$ Y. Qian, S. Orlov, G. Barhastathis, and D. Psaltis, OSA Annual Meeting '93, Technical Digest 16, TuH 4, 78 (1993). 